\title{
MÉTODOS ALTERNATIVOS DE OBTENÇÃO DE PESO VIVO EM BOVINOS DA RAÇA NELORE
}

\begin{abstract}
José Roberto da Costa Júnior ${ }^{1}$, Fernando Pereira Lima ${ }^{2}$, Eduardo Pereira Fonseca ${ }^{2}$, Mateus Lopes Fleury ${ }^{2}$, Andreza Millena Pires Ferreira ${ }^{2}$

${ }^{1}$ Professor Mestre do curso de Medicina Veterniária da UniMB, São Luís de Montes Belos-GO, Brasil. e-mail: joseroberto.costa@fmb.edu.br

${ }^{2}$ Docente do curso de Medicina Veterinária da UniMB, São Luís de Montes Belos-
\end{abstract} GO, Brasil.

\section{Recebido em: 06/04/2019 - Aprovado em: 10/06/2019 - Publicado em: 30/06/2019 DOI: 10.18677/EnciBio_2019A28}

\begin{abstract}
RESUMO
O trabalho representa o resultado de um estudo de correlações entre medidas corporais como o perímetro torácico, comprimento corporal e o peso vivo de machos da raça Nelore em período de recria, criadas na fazenda Boa Esperança, localizada no município da cidade de Goiás. O objetivo foi de verificar se haveriam diferenças entre os pesos obtidos por três métodos alternativos a balança mecânica (BA), a fim de avaliar a eficácia do emprego da fita torácica de pesagem (FT), circunferência torácica multiplicada pelo fator 2,8 (CT) e pela equação de quetelet (EQ). Foram avaliados 92 animais com idade entre 18 e 20 meses, onde realizou-se pesagens utilizando os métodos citados. Executou-se o teste Tukey, a fim de comparar uma possível variação entre as médias, sendo a primeira referente a $\mathrm{FT}$, a segunda referente a CT, a terceira obtida por EQ e BA como referência de pesagem. Notouse que houve variações nas médias $(B A=274,89, F T=301,36, C T=278,05$ e $E Q=$ $213,17)$, e desvio padrão $(B A=21,13, F T=30,47, C T=13,24$ e $E Q=24,50)$, não havendo diferença entre os métodos $\mathrm{BA}$ e $\mathrm{CT}$, porém havendo diferença entre os métodos FT e EQ. Assim, podemos assegurar que o perímetro torácico, obtido pela fita métrica multiplicado pelo fator 2,8 para avaliação do peso corporal de bovinos, é uma técnica alternativa precisa e de boa credibilidade, sendo uma ferramenta que pode ser aplicada por empresários rurais com confiança.
\end{abstract}

PALAVRAS-CHAVE: fita torácica, perímetro torácico, peso corporal.

\section{ALTERNATIVE METHODS OF OBTAINING LIVE WEIGHT IN NELORE BREED BOVINE}

\begin{abstract}
The work represents the result of a study of correlations between body measurements such as the thoracic perimeter, body length and live weight of males of the Nelore breed in the rearing period, which were raised at Boa Esperança Farm, located in the city of Goiás. was to verify if there were differences between the weights obtained by three alternative mechanical scales (BA), in order to evaluate the efficacy of the use of the thoracic weighing (FT), chest circumference multiplied
\end{abstract}


by factor 2,8 (CT) and by the quetelet equation (EQ). We evaluated 92 animals aged 18 to 20 months, where weights were performed using the methods mentioned. The Tukey test was performed in order to compare a possible variation between the averages, the first one referring to $\mathrm{FT}$, the second referring to $\mathrm{CT}$, the third one obtained by $\mathrm{EQ}$ and $\mathrm{BA}$ as weighing reference. It was observed that there were variations in the means $(\mathrm{BA}=274.89, \mathrm{FT}=301.36, \mathrm{CT}=278.05$ and $\mathrm{EQ}=213.17)$, and standard deviation ( $\mathrm{BA}=21.13, \mathrm{FT}=30.47, \mathrm{CT}=13.24$ and $\mathrm{EQ}=24.50$ ), there being no difference between the BA and CT methods, but there was a difference between the FT and EQ methods. Thus, we can assure that the thoracic perimeter, obtained by the tape measure multiplied by the factor of 2.8 to evaluate the body weight of cattle, is an accurate alternative technique of good credibility, being a tool that can be applied by rural entrepreneurs with confidence .

KEYWORDS: thoracic tape, thoracic perimeter, body weight.

\section{INTRODUÇÃO}

A pecuária brasileira tem sido uma importante produtora de alimentos nos últimos anos e se inseriu no mercado internacional como protagonista, principalmente na produção de proteína animal, sendo comercializada em quilogramas de carcaça (EUCLIDES FILHO, 2015).

Devido a grande representatividade brasileira na produção de bovinos, há a necessidade da ponderação do animal vivo, sendo importante para avaliação do crescimento, estado nutricional, administração adequada de medicamentos e parasiticidas, além de ajustes no arraçoamento, de forma a se estabelecer metas de ganho de peso, conforme o sistema de produção (PEREIRA et al., 2010).

Conhecer o desempenho de produção é uma tarefa básica do pecuarista. Afinal, é preciso saber se a criação está dando lucro ou prejuízo. Para tanto, deve ser efetuado um acompanhamento preciso de todos as etapas do sistema de produção trabalhado. Feito isso, além de ter em mãos um instrumento para tomada de decisão dentro de sua exploração, o pecuarista pode saber como reagir e se seus parâmetros estão dentro dos padrões aceitáveis para a meta tracejada (LAZZARINI NETO, 2017a).

Portanto, conhecer o peso vivo de bovinos nos diferentes sistemas de criação, é de grande importância para o produtor que almeja traçar um planejamento dentro da cria, recria ou engorda. De fato, é de confiança do pecuarista as balanças fixadas nos currais de manejo, porém, essa é uma realidade para poucos produtores, devida principalmente ao custo de implantação e manutenção do equipamento.

A balança é um equipamento indispensável ao pecuarista, já que permite ele que acompanhe com precisão o desempenho do gado em termos de ganho de peso. Há vários tipos de balança no mercado, as quais permitem pesar um ou mais bovinos de uma vez. A escolha depende das necessidades operacionais da fazenda. Existem, também, as balanças eletrônicas, que conferem alta precisão e rapidez de pesagem. (LAZZARINI NETO, 2017b).

A cada ciclo produtivo, fica mais difícil trabalhar sem informação, principalmente pela redução de margens líquidas, encarecidas pelos custos de produção, ficando difícil para o produtor tradicionalista permanecer na atividade. A tecnologia é um caminho inevitável dentro da pecuária de precisão, mas podemos utilizar de tecnologias baratas e de confiança para traçarmos estratégias nutricionais e de negócios. 
Métodos indiretos de pesagem como o uso de fita torácica e equações utilizadas por determinados pesquisadores, podem estimar o peso corporal de bovinos, porém existem variáveis nesses métodos que limitam seu uso, como por exemplo, o estresse dos animais causados no curral de manejo e posicionamento do animal em momento de coleta da informação (MAIA, 2013).

Estudos demonstram que há uma elevada correlação entre o peso vivo (PV) do animal e determinadas medidas corporais, podendo algumas destas ser utilizadas com o intuito de estimar o PV dos animais na ausência de balança para pesagem (CYRILLO et al., 2013).

Pesagens estimadas por equações são encontradas através de medidas corporais, como o perímetro torácico e o comprimento corporal (REIS et al., 2008). Quando o animal tem como destino a comercialização em frigorífico, o mesmo não terá problemas quanto ao peso, pois esse será pesado no próprio estabelecimento, através de uma balança fixa. No entanto, para se calcular dietas e até mesmo dosagem de medicamentos de bovinos, existe a necessidade de se conhecer o peso individual ou do lote, para que não haja qualquer tipo de erro, principalmente em sub ou super dosagem de medicamentos (ABREU et al., 2015).

$\mathrm{O}$ presente trabalho teve como objetivo analisar as principais alternativas ponderais encontradas na literatura aliada ao peso de balança fixa, verificando se existe diferença significativa na obtenção do peso corporal a partir da aferição com os métodos mencionados, em machos de recria da raça nelore, determinando se há alguma opção que pode ser empregada com segurança por produtores rurais.

\section{MATERIAL E MÉTODOS}

O estudo foi conduzido na fazenda Boa Esperança, localizada no município da cidade de Goiás (Latitude: -15914646, Longitude: -50,470749) estado de Goiás. Foi avaliado o peso corporal de 92 (noventa e dois) animais machos da raça Nelore em sistema de recria, com idade entre 18 e 20 meses.

Os bovinos foram conduzidos para o curral de manejo em rotina de serviço da fazenda, e ali permaneceram em jejum por 12 horas. Após esse período os animais em condições de bem estar, foram contidos de forma individual em um tronco de contenção para ser efetuado as medidas corporais (Figura 1), que foram o perímetro torácico, comprimento do corpo (ponta caudal da escápula à ponta da nádega), alem do peso obtido pela fita de pesagem. Em seguida o mesmo animal era conduzido para a balança fixa, onde ali aferia-se o peso corporal.

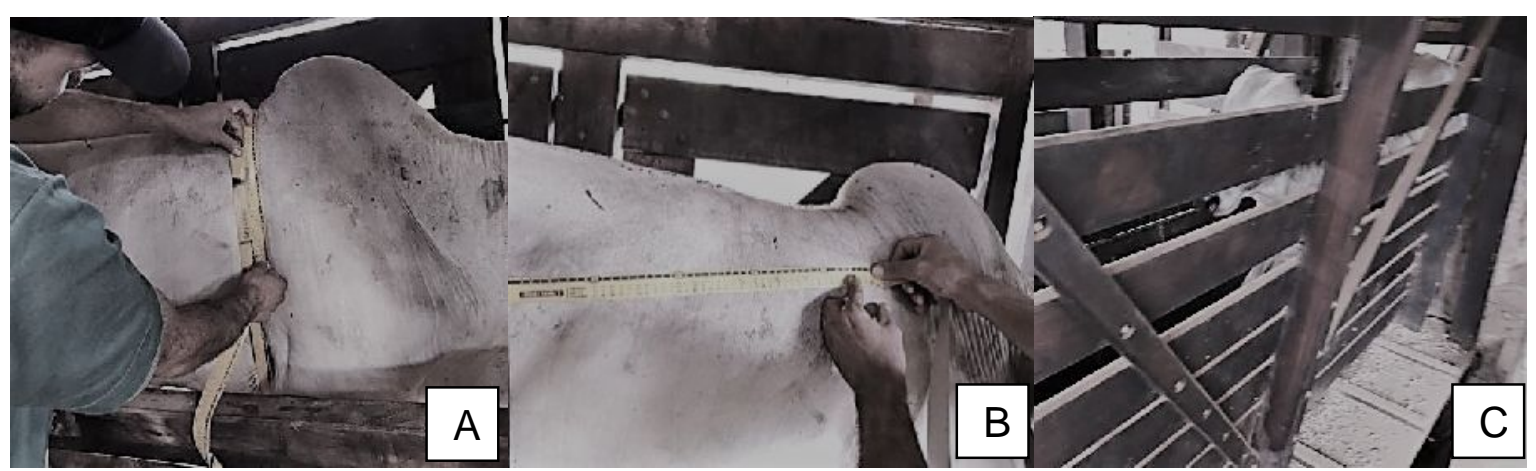

FIGURA 1 - Medidas utilizadas para coleta de informações. (A) circunferência torácica e peso obtido com fita torácica; (B) comprimento corporal; (C) Balança utilizada para pesagem. 
Ressalta-se que a tara da balança mecânica era realizada frequentemente afim de não ter interferência no resultado final e as medidas corporais eram coletadas através do brete de contenção somente quando o animal se encontrava com os quatro membros apoiados na superfície e com a posição corporal mais ereta possível, para melhor acurácia dos dados. Não houve troca ou rodízio de mão de obra durante os procedimentos na balança, fita torácica e coleta de medidas corporais, a fim de se evitar a ocorrência de variações.

Os dados obtidos pela circunferência torácica foram multiplicados pelo fator 2,8, obtendo assim o peso de CT, conforme metodologia utilizada por Abreu et al., 2015. A mesma fita foi usada para realizar a pesagem direta através da circunferência torácica, utilizando como parâmetro presente na fita, a raça média.

Para encontrar o peso pela equação de Quetelet, foi utilizado a formula $\left(\mathrm{P}=\mathrm{C}^{2} \times \mathrm{L} \times 87,5\right)$, onde: $\mathrm{P}=$ peso vivo $(\mathrm{kg}) ; \mathrm{C}=$ perímetro torácico $(\mathrm{m}) ; \mathrm{L}=$ comprimento do corpo $(\mathrm{m}) ; 87,5=$ coeficiente de ajuste, conforme Procreare, 2018. No total foram encontrados os pesos de quatro formas diferentes, sendo elas balança mecânica (BA), fita torácica $(F T)$, circunferência torácica $\times 2,8(C T)$ e equação de Quetelet (EQ).

As análises foram realizadas por meio do programa computacional de análise estatística e planejamento de experimentos Sisvar (Ferreira, 2011). Os dados foram tabulados e analisados estatisticamente, ao nível de $5 \%$ de probabilidade, utilizando para comparação de médias o teste de Tukey, para analisar as possíveis variações entre os tratamentos.

\section{RESULTADOS E DISCUSSÃO}

$\mathrm{Na}$ tabela 1, apresenta as médias de pesos corporais obtidos através da realização dos quatro métodos de pesagem, que são fita torácica (FT), comprimento torácico x $2.8(\mathrm{CT})$, balança mecânica (BA) e equação de Quetelet (EQ), onde é apresentado o desvio padrão (DP) e o coeficiente de variação (CV), analisados pelos pesos corporais dos 92 machos da raça Nelore. De acordo com os valores explicitados, nota-se que houve variações entre as médias, sendo possível afirmar que não houve diferença entre os pesos obtidos pelos métodos BA e CT, porém diferindo dos métodos FT e EQ.

TABELA 1. Médias de pesagens, desvio padrão e coeficiente de variação de acordo com os métodos utilizados para mensuração do peso vivo de animais machos nelore em sistema de recria.

\begin{tabular}{|c|c|c|c|c|c|c|}
\hline & \multicolumn{4}{|c|}{ Método de pesagem } & \multirow{2}{*}{$\begin{array}{l}\text { Média } \\
\text { geral }\end{array}$} & \multirow{2}{*}{$\begin{array}{l}\text { CV } \\
(\%)\end{array}$} \\
\hline & BA & FT & CT & $E Q$ & & \\
\hline Média $(\mathrm{kg})$ & $274,89^{a}$ & $301,36^{b}$ & $278,05^{a}$ & $213,17^{\mathrm{C}}$ & 4 & 876 \\
\hline $\mathrm{DP}(\mathrm{kg})$ & 21,13 & 30,47 & 13,24 & 24,5 & & \\
\hline
\end{tabular}

$\mathrm{BA}=$ balança mecânica; $\mathrm{FT}$ = fita toráxica; $\mathrm{CT}=$ Circunfêrencia toráxica $\times 2,8 ; \mathrm{EQ}$ = equação de quetelet; $\mathrm{DP}=$ desvio padrão; $\mathrm{CV}=$ coeficiente de variação. Médias nas linhas seguidas de letras iguais, não diferem estatisticamente entre si pelo teste de Tukey a $5 \%$ de probabilidade.

Segundo Watanabe et al. (2017), haviam diferenças entre os pesos obtidos pelo emprego da fita torácica de pesagem e da balança digital em animais da raça Nelore com idade entre 15 e 17 meses, e chegaram a conclusão de que não houve 
diferença entre os métodos comparados, o que não foi observado no presente trabalho entre as médias de BA e FT, diferindo de forma significativa.

Setim et al. (2010), também comparando fita torácica e balança, afirmaram em seu trabalho que embora houvesse uma pequena variação entre os valores obtidos entre os tratamentos, chegaram a conclusão de que a fita torácica é uma ferramenta alternativa para obtenção de peso, podendo ser empregada com segurança.

As médias dos pesos corporais obtidos entre os tratamentos BA, FT, EQ e CT (Figura 2), demonstram que entre os tratamentos BA e CT foram equiparados, com DP evidenciando baixa dispersão entre os dados em torno da média, o que nos leva a crer que podemos sim confiar nos dados do tratamento CT perante BA, o que não foi observado por Abreu at al. 2015, onde os mesmos compararam BA e CT, chegando a conclusão de que o tratamento CT não se mostrou aplicável para a amostra analisada. Para as médias observadas entre os tratamentos FT e EQ, observa-se um DP evidenciando alta dispersão entre os dados em torno da média, o que se refere à grande variação entre os pesos obtidos individualmente entre os animais dos respectivos tratamentos.

$\square \mathrm{PV} \square \mathrm{DP}$

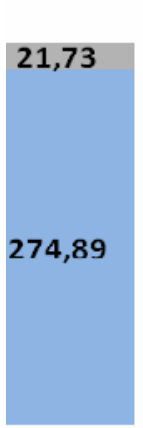

BA

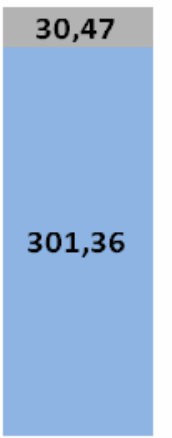

FT

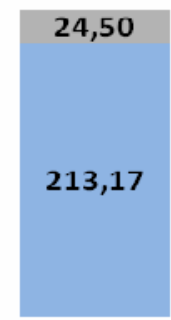

$\mathrm{EQ}$

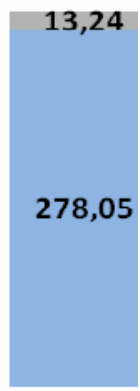

CT

FIGURA 2: Média do peso vivo (PV) e desvio padrão (DP) de bovinos machos Nelore não castrados com o uso da balança mecânica (BA), fita torácica de pesagem (FT), equação de quetelet (EQ) e através do uso da fita métrica comum (valor obtido multiplicado por 2,8$)(\mathrm{CT})$.

Observou-se que as médias dos resultados obtidos pelos métodos de mensuração de peso vivo de animais nelores, apresentaram variações insignificantes exceto na EQ e FT, onde se constou estatisticamente uma variação considerável se comparada aos outros métodos. As médias de CT e BA representam respectivamente $278,05 \mathrm{~kg}, 274,89 \mathrm{~kg}$. Enquanto a média de EQ apresentou 213,17 kg e FT 301,36 kg.

De acordo com Abreu et al. (2015) e Watanabe et al. (2017), demonstraram que a fita torácica tem acurácia em sua utilização e precisão do peso estimado do animal, servindo como um método indireto e alternativo de valor relativamente baixo se comparado a balança mecânica ou digital. Segundo Setim et al., (2010) afirmaram em seu trabalho que embora houvesse uma pequena variação entre os valores obtidos da fita torácica e a balança, os autores concluíram que a fita torácica é uma excelente ferramenta para obtenção de peso e pode ser empregada com segurança. 


\section{CONCLUSÃO}

O perímetro torácico obtido pela fita métrica mostrou eficiência e boa credibilidade na mensuração do peso de animais da raça Nelore em jejum, sendo uma ferramenta de fácil manuseio na presença de tronco de contenção e acessível a produtores de pequenas propriedades, encaixando-se como um meio alternativo e indireto na obtenção de peso corporal.

\section{AGRADECIMENTOS}

A UniMB por nos proporcionar a oportunidade de aprendizado e ao GEBOV (Grupo de Estudos em Bovinocultura), por estar sempre buscando respostas as dificuldades encontradas pelos produtores rurais a nível de campo.

\section{REFERÊNCIAS}

ABREU, B.A.; MAGALHAES, C.J.; DUAYER, E.; MCHADO, S.H.M.; SILVA, D.A.; Variação da medida torácica obtida com a fita métrica tradicional com fator de correção e com a fita de pesagem para bovinos. Acta Biomedica Brasiliensia, v. 6, n. $2, \quad$ p. $\quad 42-48, \quad 2015 . \quad$ Disponível em: http://www.actabiomedica.com.br/index.php/acta/article/view/19. DOI: http://dx.doi.org/10.18571/acbm.084.

CYRILLO, J.N.S.G.; MERCADANTE, M.E.Z.; BONILHA, S.F.M. Relações entre medidas biométricas, características de carcaça e cortes cárneos comerciais em bovinos Zebu e Caracu. CAPTA; APTA; PRDTA. Artigos Técnicos, 2013. Disponível em: http://pt.engormix.com/MA-pecuaria-corte/frigorifico/artigos/relacoesentre-medidas-biometricas-t1523/378-p0.htm Acesso em: 04 de jan 2019.

EUCLIDES FILHO, K.. Bovinocultura de corte no Brasil. Revista de Política Agrícola, Brasília-DF, 16, Jun. 2015. Disponível em: $<$ https://seer.sede.embrapa.br/index.php/RPA/article/view/495>. Acesso em: $28 \mathrm{Fev}$. 2019.

FERREIRA, D.F.;. Sisvar: a computer statistical analysis system. Ciência e Agrotecnologia (UFLA), v. 35, n.6, p. 1039-1042, 2011. DOI: 10.1590/S141370542011000600001.

FERRO, R. A. C; Predição do peso vivo de bovinos em função da altura da cernelha e do perímetro torácico. IN: III Encontro de Pesquisa e Extensão e Semana Nacional de Ciência e Tecnologia São Luis de Montes Belos, 2015. Disponível em:<http://www.anais.ueg.br/index.php/epe-s/mb/article/view/3329>. Acesso em: 18 de out. de 2018.

LAZZARINI NETO, S. Lucrando com a pecuária. In: ALHADAS, H. M., DUARTE, M. S. Cria e recria na pecuária de corte. 4 ed. Viçosa: Aprenda fácil, 2017a. $160 \mathrm{p}$.

LAZZARINI NETO, S. Lucrando com a pecuária. In: ALHADAS, H. M., DUARTE, M. $S$. Instalações e benfeitorias na pecuária de corte. 3 ed. Viçosa: Aprenda fácil, 2017b. 133 p. 
MAIA, P.V. Monitoramento do ganho de peso diário em bezerras e novilhas. Rehagro, Artigos Técnicos, 2013. Disponível em: http://rehagro.com.br/plus/modulos/noticias/ler.php? cdnoticia=2605. Acesso em 12 de dez 2018.

PEREIRA, E. S., PIMENTEL, P. G., QUEIROZ, A. C. \& MIZUBUTI, I. Y. 2010. Novilhas leiteiras. Graphiti Gráfica e Editora Ltda, Fortaleza, Ceará.

REIS, G. L.; ALBUQUERQUE, F.H.M.A.R.; VALENTE, B.D.; MARTINS, G.A.; TEODORO, R.L.; Predição do peso vivo a partir de medidas corporais em animais mestiços Holandês/Gir. Ciência Rural, v. 38, n. 3, p. 778-783, 2008. Disponível em: https://www.redalyc.org/pdf/331/33138329.pdf. Acesso em: 18 de dez. de 2018.

ROCREARE. Como pesar seu gado sem balança. 2018. Disponível em: http://procreare.com.br/como-pesar-o-gado-sem-balanca/. Acesso em: 18 de dez. de 2018.

SETIM, D.H.; VANZAN, M.; FERNANDES, M.V.; Comprovação da eficácia do uso da fita torácica de pesagem em bovinos leiteiros. 2010. Disponível em: http://www.cafw.ufsm.br/mostraciencias/2011/resumos/210.pdf Acesso em 04 de nov. 2015.

WATANABE, A.H. Q.;. MANCHINI, H.; MARÇAL, W.S.; Comparação do peso corporal obtido através de pesagem em balança digital ou fita torácica de pesagem em nelores machos. PUBVET, v. 11, p. 424-537, 2017. Disponível em: http://www.pubvet.com.br/artigo/3758/comparaccedilatildeo-do-peso-corporalobtido-atraveacutes-de-pesagem-em-balanccedila-digital-ou-fita-toraacutecica-depesagem-em-nelores-machos. doi: 10.22256/PUBVET.V11N5.527 - 531. 